\title{
Optimized DNA sampling of ancient bones using Computed Tomography scans
}

\author{
Federica Alberti ${ }^{1}$ (D) | Javier Gonzalez ${ }^{1}$ | Johanna L. A. Paijmans ${ }^{1}$ | Nikolas Basler ${ }^{1}$ | \\ Michaela Preick $^{1}$ | Kirstin Henneberger ${ }^{1}$ | Alexandra Trinks ${ }^{1,2}$ | Gernot Rabeder ${ }^{3}$ | \\ Nicholas J. Conard ${ }^{4}$ | Susanne C. Münzel ${ }^{4}$ | Ulrich Joger ${ }^{5}$ | Guido Fritsch ${ }^{6}$ | \\ Thomas Hildebrandt $^{6}$ | Michael Hofreiter ${ }^{1}$ | Axel Barlow ${ }^{1}$
}

${ }^{1}$ Institute for Biochemistry and Biology, University of Potsdam, Potsdam, Germany

${ }^{2}$ Evolutionary Biology, IRI for the Life

Sciences, Humboldt University Berlin,

Berlin, Germany

${ }^{3}$ Department of Palaeontology, Geozentrum, University of Vienna, Vienna, Austria

${ }^{4}$ Institute for Archaeological Sciences, Archaeozoology, University of Tübingen,

Tübingen, Germany

${ }^{5}$ Staatliches Naturhistorisches Museum Braunschweig, Braunschweig, Germany

${ }^{6}$ Leibniz Institute for Zoo and Wildlife

Research, Berlin, Germany

\section{Correspondence}

Thomas Hildebrandt, Leibniz Institute for Zoo and Wildlife Research,

Forschungsverbund Berli e.V., Alfred-

Kowalke-Str. 17, 10315 Berlin, Germany.

Email: hildebrand@izw-berlin.de

and

Michael Hofreiter and Axel Barlow, Institute for Biochemistry and Biology, University of Potsdam, 14476 Potsdam, Germany.

Emails: michi@palaeo.eu (M. H.); axel.barlow. ab@gmail.com (A. B.)

Funding information

ERC consolidator grant GeneFlow, Grant/ Award Number: 310763; Klaus Tschira foundation, Grant/Award Number: 00.298 .2016

\begin{abstract}
The prevalence of contaminant microbial DNA in ancient bone samples represents the principal limiting factor for palaeogenomic studies, as it may comprise more than $99 \%$ of DNA molecules obtained. Efforts to exclude or reduce this contaminant fraction have been numerous but also variable in their success. Here, we present a simple but highly effective method to increase the relative proportion of endogenous molecules obtained from ancient bones. Using computed tomography (CT) scanning, we identify the densest region of a bone as optimal for sampling. This approach accurately identifies the densest internal regions of petrous bones, which are known to be a source of high-purity ancient DNA. For ancient long bones, CT scans reveal a high-density outermost layer, which has been routinely removed and discarded prior to DNA extraction. For almost all long bones investigated, we find that targeted sampling of this outermost layer provides an increase in endogenous DNA content over that obtained from softer, trabecular bone. This targeted sampling can produce as much as 50 -fold increase in the proportion of endogenous DNA, providing a directly proportional reduction in sequencing costs for shotgun sequencing experiments. The observed increases in endogenous DNA proportion are not associated with any reduction in absolute endogenous molecule recovery. Although sampling the outermost layer can result in higher levels of human contamination, some bones were found to have more contamination associated with the internal bone structures. Our method is highly consistent, reproducible and applicable across a wide range of bone types, ages and species. We predict that this discovery will greatly extend the potential to study ancient populations and species in the genomics era.
\end{abstract}

KEYWORDS

ancient DNA, computer tomography, palaeogenomics, paleogenetics, petrous bone

\section{1 | INTRODUCTION}

The accumulation of contaminant, exogenous DNA in ancient biological remains represents a universal challenge in the field of palaeogenomics. Levels of contaminant microbial DNA may frequently exceed $99 \%$ of molecules obtained from ancient samples, which incurs a directly proportional increase in sequencing costs relative to high-quality modern tissues. As a result, huge sequencing 
efforts may be required to obtain even modest amounts of endogenous sequence data (Meyer et al., 2016; Noonan et al., 2005; Orlando et al., 2013), which imposes practical and financial limits on the study of palaeogenomes.

Several approaches are available to increase the fraction of endogenous DNA obtained from ancient samples. Hybridization capture allows the selective removal of contaminant DNA, producing a relative enrichment of sequencing libraries for specific genomes (Enk et al., 2014; Paijmans, Fickel, Courtiol, Hofreiter, \& Förster, 2016) or genomic regions (Castellano et al., 2014). This method has several limitations, however, requiring either prior DNA sequence knowledge of the region(s) of interest (Gnirke et al., 2009; Hodges et al., 2007), or high-quality DNA from an extant close relative (Enk et al., 2014). Hybridization capture may also produce biases towards particular genome regions and repeat elements (Enk et al., 2014), and may require complex optimization of laboratory methods (Paijmans et al., 2016) to ensure experimental success. A second approach for increasing the fraction of endogenous DNA is chemical treatment of bone powder to selectively destroy contaminant DNA molecules. Such methods include predigestion with proteinase $\mathrm{K}$ (Damgaard et al., 2015; Gamba et al., 2016; Clio Der, Balanovsky, Templeton, \& Llamas, 2014) and pretreatment with phosphate buffer or sodium hypochlorite (Korlević et al., 2015). However, all pretreatment approaches are associated with a parallel loss in endogenous DNA which is frequently dramatic (Basler et al., 2017; Korlević et al., 2015). Sample pretreatment may also be unpredictable (Basler et al., 2017) and has even been contra-indicated for samples with very limited availability of bone material (Korlević et al., 2015). A third approach for increasing endogenous content is to utilize laboratory methods that target specific DNA fragment sizes, as reported for DNA extraction (Dabney et al., 2013; Glocke \& Meyer, 2017) and some library preparation methods (Bennett et al., 2014). These increases presumably reflect substantial differences in the fragment length distributions of endogenous and contaminant DNA. However, such approaches are likely to be sample-specific and difficult to apply in any predictable way without detailed prior knowledge of sample properties and metagenomic composition. Finally, an approach based on selective enrichment of molecules containing uracil residues, which accumulate in ancient DNA fragments as a result of postmortem damage, has been developed (Gansauge \& Meyer, 2014). However, this method leads to the loss of undamaged ancient molecules and is also ineffective in reducing the proportion of ancient contaminant molecules.

The simplest and potentially most effective approach for increasing the relative endogenous DNA fraction is to target specific bones or skeletal regions that are associated with low levels of contamination. A widespread practice in ancient DNA research is to remove the outermost bone layer (Damgaard et al., 2015; Fortes et al., 2016; Hansen et al., 2017; Rohland \& Hofreiter, 2007) as this is assumed to contain greater levels of contamination than internal bone regions, which are not directly exposed to the external environment. To our knowledge, this hypothesis has not been empirically tested in any rigorous way, however. Further progress in targeted sampling approaches has been achieved recently, including the discovery of the mammalian petrous bone as a source of exceptionally pure endogenous DNA (Gamba et al., 2014; Pinhasi et al., 2015), and the cementum tooth layer (Damgaard et al., 2015; Hansen et al., 2017), which provides substantial increases in endogenous DNA content over dentine. Although these discoveries represent major advances in the study of palaeogenomics, no currently available targeted sampling approach provides a consistent, reproducible and effective increase in endogenous DNA that can be applied across all vertebrate taxa and bone types.

A notable outcome of previous studies is that bone density and endogenous DNA content seem to be positively correlated (Pinhasi et al., 2015). Thus, in general, sampling of denser bone regions may provide a basis for increasing endogenous DNA recovery from ancient bone samples. Computed tomography (CT) imaging is a widely used method for accurately measuring density variation in biological tissues. It has been regularly applied in the fields of archaeology and palaeontology as a noninvasive method to determine a three-dimensional image of the external and internal structure of objects. In the case of subfossil bones, CT-based bone biopsy and reconstruction can contribute unique information about extinct species, for example the morphometrics of internal cavities, palaeopathology (Cramer, Brix, Matin, Rühli, \& Hussein, 2017; Griffin, Rawlinson, McDonald, \& Duncan, 2016; lurino, Danti, Sala, \& Sardella, 2013; Lautenschlager, 2016; Väre et al., 2016) and cause of death (Kappelman et al., 2016). Furthermore, combining the three-dimensional imaging CT with 3D-printing techniques allows for restoration and digitization, and thus immortalization, of fossils (Lautenschlager, 2016).

We hypothesized that CT scanning may provide a method for targeted sampling of ancient bones to enhance endogenous DNA content. We investigated this hypothesis by CT scanning a variety of ancient bones. We found that this is an effective method of identifying the location of the otic capsule, the densest and least contaminated part of the petrous bone, allowing precise sampling even if the inner ear morphology of the investigated species is not well known. In addition, we identify a high-density outermost bone layer that surrounds the midshaft in long bones. We find that targeted sampling of this outermost layer provides an increase in endogenous DNA relative to other, less dense bone regions. Our method for increasing endogenous DNA recovery is simple, reproducible, effective, and does not lead to reductions of library complexity or overrepresentation of repetitive genomic elements associated with some other methods. CT scanning therefore represents a valuable and important tool for future studies of palaeogenomes.

\section{MATERIALS AND METHODS}

\subsection{CT scanning of ancient bones}

We first investigated the potential for CT scans in locating the previously identified optimal region (otic capsule) of the petrous bone (Pinhasi et al., 2015). Our ongoing work of sampling the petrous bones of a variety of mammal species has shown that identification 
of the otic capsule can be challenging, and a method to achieve this that minimizes potential damage to precious palaeontological specimens is desirable. Moreover, diagenesis may result in certain parts of the otic capsule being denser and therefore more suitable for DNA extraction. We also investigated the potential for CT scanning to target high-density regions of (nonpetrous) long bones using eight Late Pleistocene cave bear (Ursus spelaeus complex) and three Late Pleistocene leopard (Panthera pardus) subfossil samples (Table 1). Complete details of all samples and specimens used in this study are shown in Table 1.

Scans were made with a clinical CT scanner Aquilion ONE from Toshiba Medical, Japan. Bone samples were scanned in $0.5-\mathrm{mm}$ slices and a data set of $0.25-\mathrm{mm}$ slices was calculated. The scan parameters were $120 \mathrm{kV}$ at $300 \mathrm{~mA}$ and a rotation time of $0.5 \mathrm{~s}$ or $1 \mathrm{~s}$ per rotation. Postprocessing of image data included measurement of bone density using different modalities.

\subsection{Bone sampling}

Based on the results of CT scans, we compared the properties of DNA extracted from bone regions of high and low densities, identified by bright and darker regions of the CT images, respectively. Details of the sampled regions are provided in Table 2. For petrous bones, two sampling treatments were investigated, the otic capsule and trabecular (spongy) bone representing, respectively, the highest and lowest density regions of the petrous bone samples. Bone powder was sampled by low speed drilling using a Dremel Fortiflex (9100-21) and a 2.4- to 2.8-mm-diameter drill bit, collecting $50 \mathrm{mg}$ of bone powder. For long bones, samples were similarly taken from the outermost bone layer and trabecular bone, representing, respectively, the highest and lowest density regions of these bones. Neither of these long bone sampling methods are typically used in ancient DNA research. We therefore additionally analysed data from seven of the long bones which had been generated previously using what we consider to be a more conventional sampling method. This involved either removal of the outermost bone layer using an abrasive rotating disc and excision of a piece of the underlying cortical (compact) bone using a core drill, followed by further cleaning by rotating disc (four cave bear samples), or removal of the outer bone layer by scratching with a sterile scalpel and excision of cortical bone by core drill (three leopard samples). The excised bone pieces were then ground to a fine powder using a pestle and mortar. Samples obtained using this conventional method have densities that are intermediate between the outermost layer and the trabecular bone.

\section{3 | DNA extraction, library preparation and sequencing}

All DNA extractions were carried out using $50 \mathrm{mg}$ of bone powder, following the protocol of Dabney et al. (2013), with reduced centrifugation speeds as described in Basler et al. (2017). One microlitre of each $25 \mu \mathrm{l}$ extract was used for quantification using a Qubit 2.0 fluorometer with high sensitivity reagents (Thermo Fisher Scientific) and verified using both positive and negative controls. DNA extracts were treated with Uracil-DNA glycosylase to remove uracil residues, which typically occur at high frequency in ancient DNA as a result of cytosine deamination, and endonuclease VIII to cleave DNA strands at abasic sites, which would otherwise block polymerase extension. DNA extracts were then converted into Illumina sequencing libraries using a single-stranded approach described in Gansauge and Meyer (2013), with a reduced concentration of Circligase II as described in Basler et al. (2017). The optimal number of library amplification PCR cycles was determined in advance using qPCR, as described in Gansauge and Meyer (2013), replicating the procedure reported in Basler et al. (2017). Indexing PCR was then performed in a reaction volume of $80 \mu \mathrm{l}$, using $20 \mu \mathrm{l}$ template library, Accuprime Pfx DNA polymerase and tailed primers to generate dual-indexed library molecules. Final library concentration and length distribution were determined using Qubit 2.0 and 2200 TapeStation (Agilent Technologies) assays, respectively. Libraries were then sequenced on the Illumina NextSeq 500 sequencing platform producing 75-bp single-end reads, following the procedures described in Paijmans et al. (2017).

\subsection{Comparison of sequence data obtained from different bone regions}

We compared four relevant properties of data obtained using different sampling treatments. These were endogenous DNA content, median fragment length, cytosine deamination and human contamination.

\subsection{1 | Endogenous DNA content}

The endogenous content of bone powder obtained using different sampling treatments was estimated by calculating the fraction of reads mapping to the reference genome assembly of a related species (Ursus maritimus and Panthera tigris altaica for cave bear and leopard sample data, respectively). Raw reads were trimmed using cutadapt v1.10 (Martin, 2011) with minimum overlap of one nucleotide, discarding any reads $<30 \mathrm{bp}$ after trimming. The processed reads were then mapped to the appropriate reference genome assembly using the bwa v0.7.8 "aln" algorithm (Li \& Durbin, 2009) with default parameters. The resulting alignment was filtered for mapping quality $(Q \geq 30)$, sorted by read position and potential $P C R$ duplicates removed using SAMtools v0.1.19 (Li et al., 2009; Supporting Information Table S1). The endogenous DNA content was estimated by the fraction of reads successfully mapping to the reference genome divided by the total number of reads used as input for mapping. Total endogenous data yield was further compared by calculating the fraction of mapped nucleotides. We also tested for potential biases introduced by our data processing pipeline by testing an alternative set of trimming and mapping parameters. These were aimed at ensuring zero carry-over of adapter sequence, ensuring only fragments sequenced in their entirety were used for mapping, and increasing the probability of mapping damaged reads (see Supporting Information Table S2). 


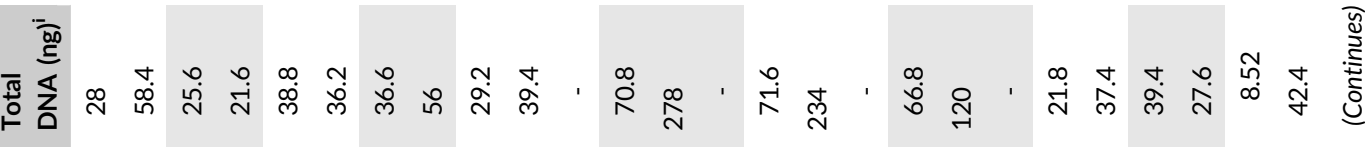

$\frac{\sqrt{2}}{\frac{\pi}{2}}$

产

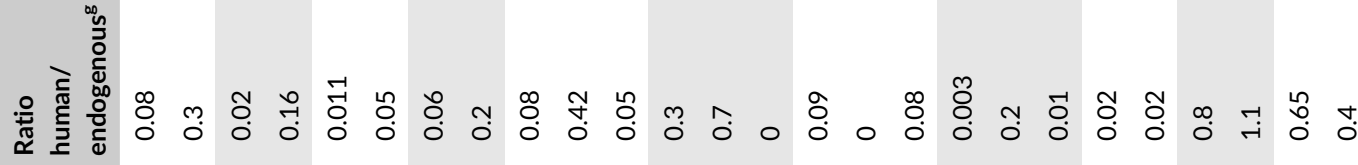

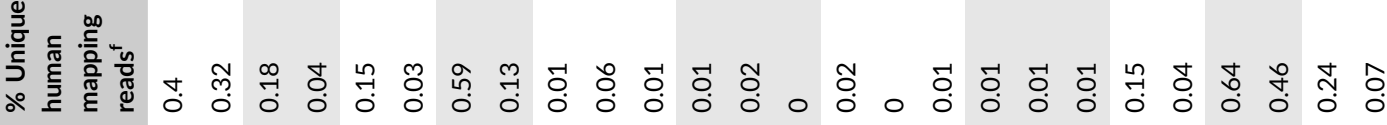

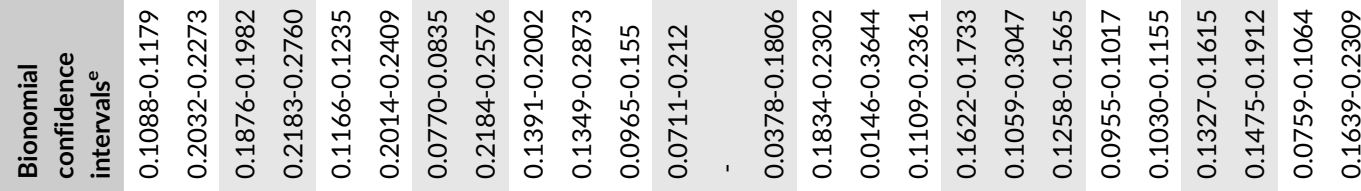

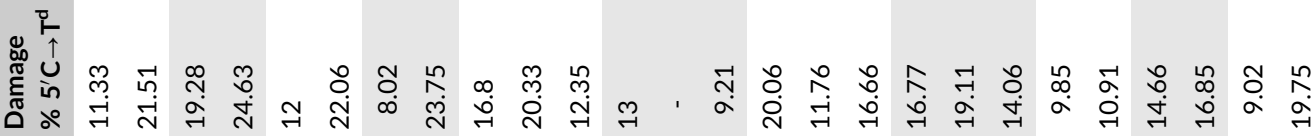

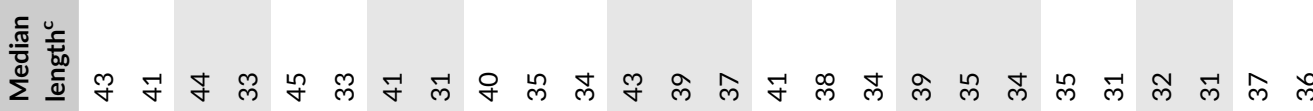

竞

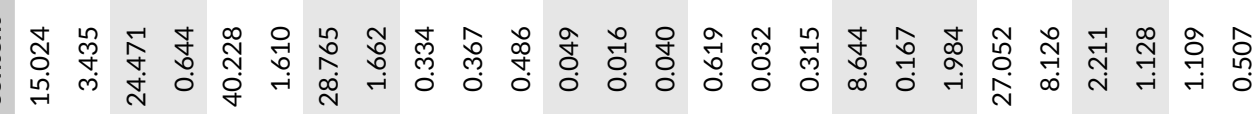

is

굴

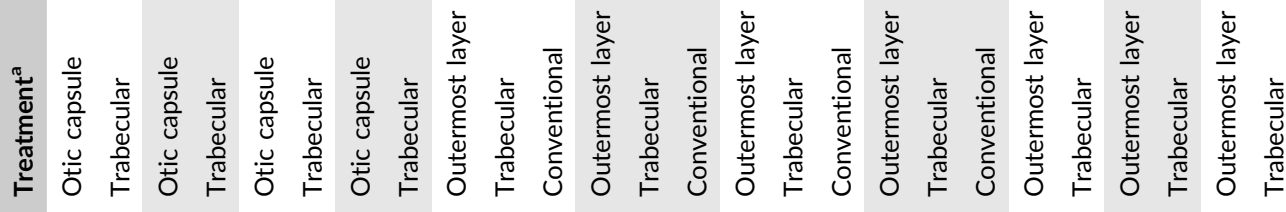

$\stackrel{n}{\underline{\underline{F}}}$

ఫ

.0.0

$\frac{\tilde{c}}{\frac{0}{\varepsilon}}$

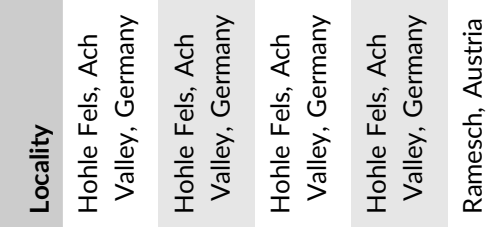

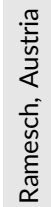

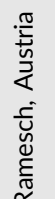

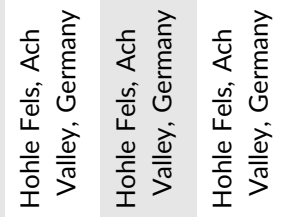

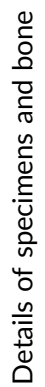

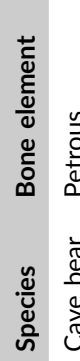

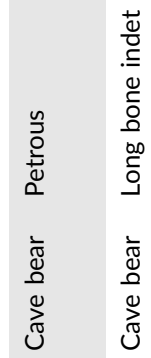

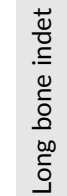

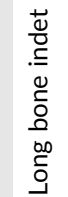

+
$\frac{0}{0}$
.5
0
0
0
0
0
0

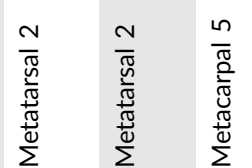

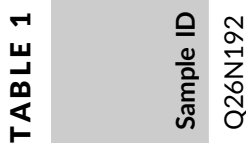

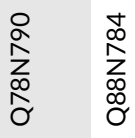

No
ż
ơ
ò

离

㝘

亥

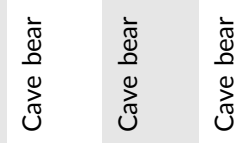

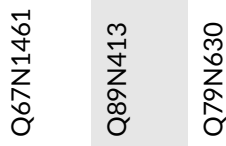



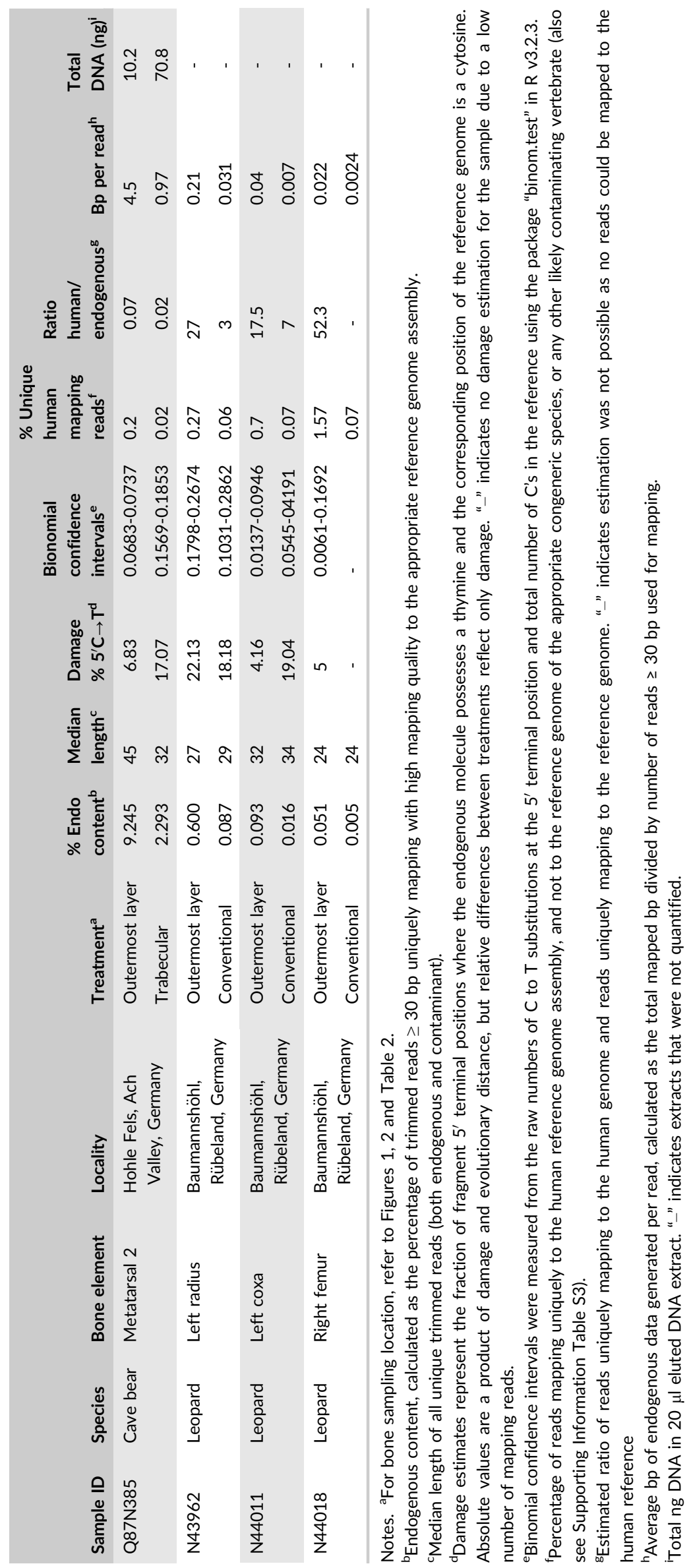
TABLE 2 Sampling strategies used for each bone treatment

\begin{tabular}{|c|c|}
\hline Treatment & Sampling strategy \\
\hline Otic capsule & $\begin{array}{l}\text { Identify the otic capsule using the CT scan and drill } \\
\text { directly into it }\end{array}$ \\
\hline $\begin{array}{l}\text { Outermost } \\
\text { layer }\end{array}$ & $\begin{array}{l}\text { The thickness of this outermost layer is } 1-1.5 \mathrm{~mm} \text {, } \\
\text { at most. Drill shallow holes into the surface of the } \\
\text { bone of a depth approximating the thickness of the } \\
\text { dense outermost layer identified by the CT images, } \\
\text { and collect the resulting powder }\end{array}$ \\
\hline Trabecular & Drill directly into the trabecular bone \\
\hline Conventional & $\begin{array}{l}\text { Remove the outer bone layer using an abrasive } \\
\text { rotating disc and excise a piece of the underlying } \\
\text { compact bone using a core drill, and proceed with a } \\
\text { further cleaning using the rotating disc, or remove } \\
\text { the outer bone layer by scratching with a sterile } \\
\text { scalpel and excise a compact bone piece by core } \\
\text { drill }\end{array}$ \\
\hline
\end{tabular}

\subsection{2 | Median fragment length}

For estimating the fragment length distribution of the total molecules obtained from each bone sample (both endogenous and contaminant), adapter trimming was carried out as described above, but without removal of short reads, then only trimmed reads were considered for length estimations. These were de-duplicated by sequence matching and removed using Tally v14-020 (Davis, van Dongen, Abreu-Goodger, Bartonicek, \& Enright, 2013), prior to length analysis.

\subsection{3 | Cytosine deamination}

A high frequency of cytosine deamination, particularly affecting the terminal ends of ancient DNA fragments, is used for both data authentication (Jónsson, Ginolhac, Schubert, Johnson, \& Orlando, 2013) and for the targeted recovery of ancient DNA molecules (Gansauge \& Meyer, 2014). We therefore investigated whether rates of cytosine deamination differed between bone sampling treatments. Although the use of Uracil-DNA glycosylase to remove uracils resulting from cytosine deamination will dramatically reduce absolute estimates, methylated cytosines deaminate to thymines and will still be represented in the resulting ancient DNA sequences data as $\mathrm{C} \rightarrow \mathrm{T}$ substitutions (Briggs et al., 2010). Our experimental design thus allows for relative comparison of deamination rates between sampling treatments, but not for absolute estimates. Cytosine deamination rates were estimated with mapDamage 2.0 (Jónsson et al., 2013). Only samples providing more than 300 mapped reads were included in the damage pattern estimation. Additionally, we only considered rates of $5^{\prime}$ deamination, as the terminal $3^{\prime}$ nucleotide of single-end reads may not always represent the fragment end.

\subsubsection{Human contamination}

Estimating human contamination by mapping reads to the reference human genome assembly could be misleading because a proportion of endogenous (cave bear or leopard) reads are likely to map to conserved regions of the human genome. In order to control for this, we mapped reads to multiple reference genome assemblies, including human, polar bear, domestic cat and a variety of other likely contaminating vertebrate species using FastQscreen v0.4.4 (Andrews, 2011) with default parameters (Supporting Information Table S3). To obtain relative estimates of human contamination, we then compared the proportion of reads uniquely mapping to the human reference genome assembly and not to any other reference genome. Although this method will not produce absolute estimates of human contamination, since many human sequences potentially also map to the genome assemblies of other mammals, it does allow relative comparisons between sampling treatments, as well as an estimation of the ratio of endogenous to human contaminant DNA. The latter was estimated by dividing the proportion of reads mapping uniquely to the human and to the correct (polar bear or tiger) reference genome.

\section{3 | RESULTS}

\subsection{Identification of the otic capsule in ancient petrous bones}

Computed tomography scanning of four Late Pleistocene cave bear petrous bones clearly identified the high-density otic capsule (Figure 1). We compared the properties of bone powder sampled from the otic capsule as identified using CT scanning with that sampled from a trabecular region of the same petrous bone. In line with previous studies (Gamba et al., 2014; Pinhasi et al., 2015), we found that the otic capsule provides an increase in endogenous content relative to trabecular bone, ranging from 4.4-fold up to 38-fold (Figure 2a, b). Endogenous DNA fragments sampled from the otic capsule are, on average, longer than those sampled from the trabecular bone (Figure 2c). As a result, increases measured in terms of endogenous nucleotide recovery are even higher than suggested by increases in endogenous molecules, ranging from 4.6-fold to 46-fold increase relative to trabecular bone regions (Figure $2 b$ ). Endogenous DNA fragments sampled from the otic capsule also showed lower levels of cytosine deamination than fragments sampled from the trabecular bone in all cases (Figure 2d). Estimated levels of human contamination were, however, higher in DNA sampled from the otic capsule (Figure 2e), but the ratio of human to endogenous reads was lower for the otic capsule in all cases due to the large increases in the proportion of endogenous DNA (Table 1). Absolute numbers of endogenous molecules in DNA sampled from the otic capsule were also higher than for trabecular bone, even in samples where the trabecular bone sample gave higher DNA quantities overall (Figure 2f, Table 1).

\section{2 | CT scanning of long bones}

For all long bones investigated, we observed a superficial high-density outer layer of the cortical bone (Figure 3a). The thickness of this outermost layer is $1-1.5 \mathrm{~mm}$, at most. To target this layer, we drilled 


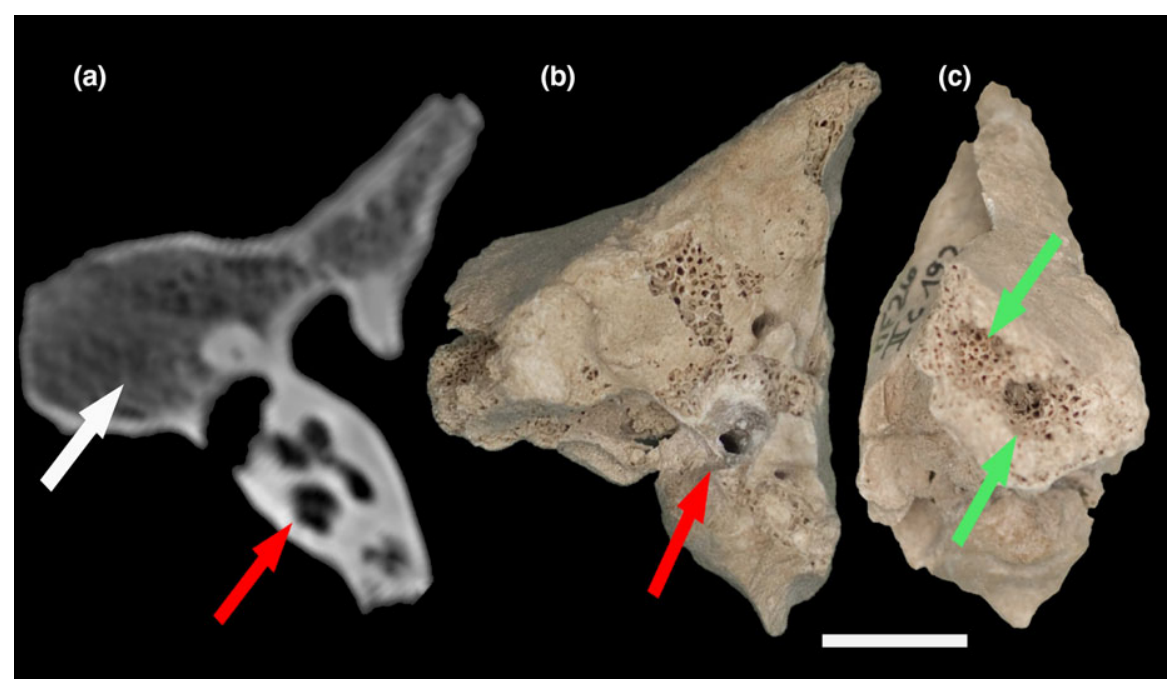

FIGURE 1 Petrous bone sampling. (a) CT scan image showing differences in the density of petrous bone tissues. Areas with higher density (red arrow) appear brighter than areas with lower density (white arrow). The two target sampling regions are indicated by arrows in panel $b$ and $\mathrm{c}$, in red for the otic capsule and in green for the trabecular bone. (b) Photograph showing the dorsal view of the petrous bone and the location of otic capsule sampling, which was performed by drilling directly into the otic capsule (red arrow). (c) Photograph showing the axial view of the petrous bone and the sampling location of the trabecular bone, which involved drilling into easily accessible trabecular area. The scale bar represents $1 \mathrm{~cm}$

shallow holes into the surface of the bone of a depth approximating the thickness of the dense outermost layer identified by the CT images and collected the resulting powder (Figure 3 b,c).

\subsection{Endogenous DNA recovery from long bone samples}

For seven of eight cave bear long bones, sampling of bone powder from the outermost layer provided an increase in endogenous DNA content relative to that sampled from trabecular bone (Figure 4a,b). The increase in endogenous DNA content ranged from 1.23-fold to 52-fold (Figure 4a,b). The largest observed fold increase equates to a change in overall endogenous DNA content from $0.17 \%$ to $8.6 \%$ (Sample SP350, Table 1). The overall pattern of increased endogenous content provided by the outermost layer is also recovered when the alternative data processing pipeline is utilized (Supporting Information Table S2).

Although sampling of the outermost layer resulted in lower total DNA recovery for all but one sample, due to the increase in endogenous DNA content, the outermost layer provided an increase in absolute endogenous DNA recovery compared to the trabecular bone for four of eight samples (Figure 4g). Notably, for the sample providing the greatest increase in endogenous DNA content, sampling of the outermost layer provided a 29-fold increase in absolute endogenous DNA recovery relative to sampling the same mass of bone powder from the trabecular bone.

Comparing results obtained from samples of the outermost layer with those obtained using conventional sampling also showed a general improvement in endogenous DNA recovery. For three of four cave bear bones, and for all three leopard bones, sampling of the outermost layer provided a relative increase in endogenous DNA recovery over conventional sampling (Figure 4a,b). This increase ranged from 1.23-fold to 9.25-fold, with the largest observed fold increase equating to a change in overall endogenous DNA content from $0.005 \%$ to $0.05 \%$ (Table 1 ).

\subsection{Human contamination of long bone samples}

A potential drawback of sampling the outermost layer is that the external surface of bones may be associated with excessive contamination from human handling which may confound downstream analyses. Of the eleven sampled long bones for which a comparison was possible (Table 1), eight showed increased human contamination associated with the outermost layer. However, for one sample contamination levels were constant across sampling treatments, and for the remaining two samples, the highest levels of human contamination were found in the trabecular bone. This trend is the same both for the absolute percentage of reads uniquely mapping the human genome, as well as the ratio of human to endogenous reads (Table 1, Figure 4f, Supporting Information Table S3). It is notable that the sample providing the highest observed fold increase in endogenous DNA content of the outermost layer relative to the trabecular bone showed no detectable change in the percentage of reads mapping uniquely to the human genome (Figure 4f, Supporting Information Table S3).

\subsection{Long bone DNA fragmentation and damage}

For all comparisons, the increase in endogenous content provided by the outermost layer of long bones is highly correlated with the increase in endogenous nucleotide recovery (Figure 4c), suggesting no large-scale differences in average endogenous DNA fragment 
(a) \% endogenous DNA content

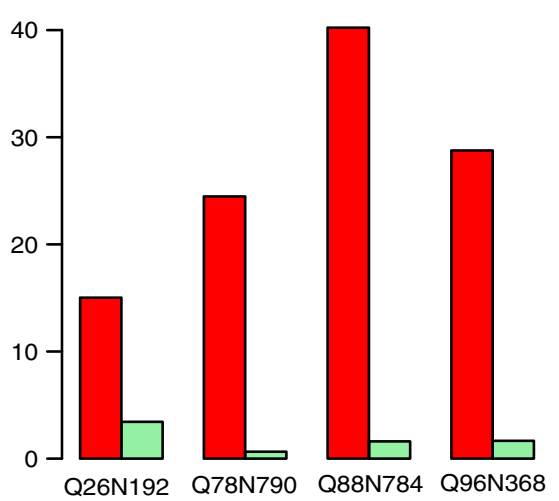

(d) Damage vs. Endo content

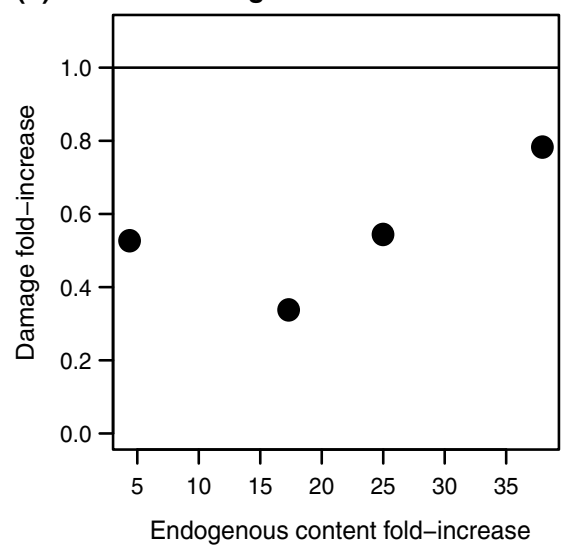

(b)

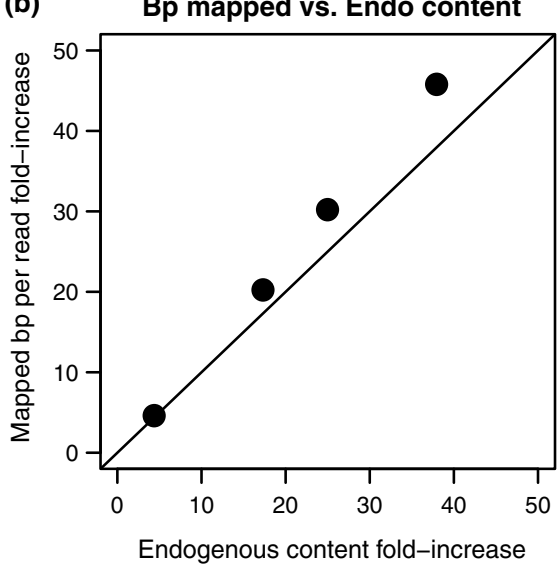

(e) Human vs. Endo content

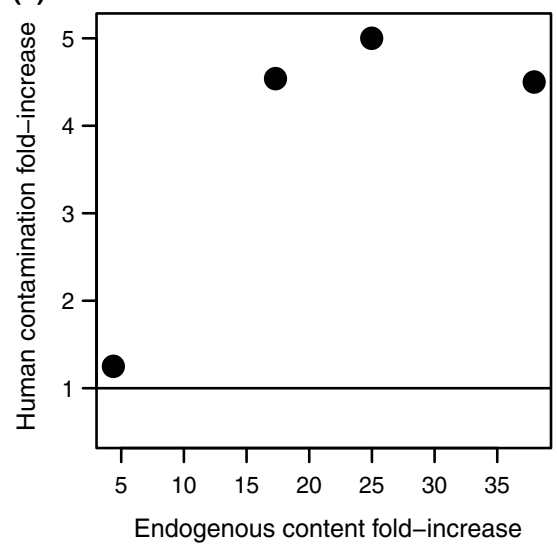

(c) Median length vs. Endo content

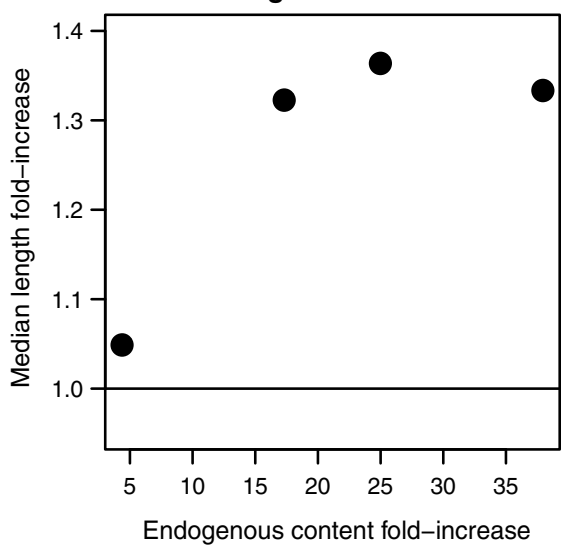

(f) Absolute DNA vs. Endo content

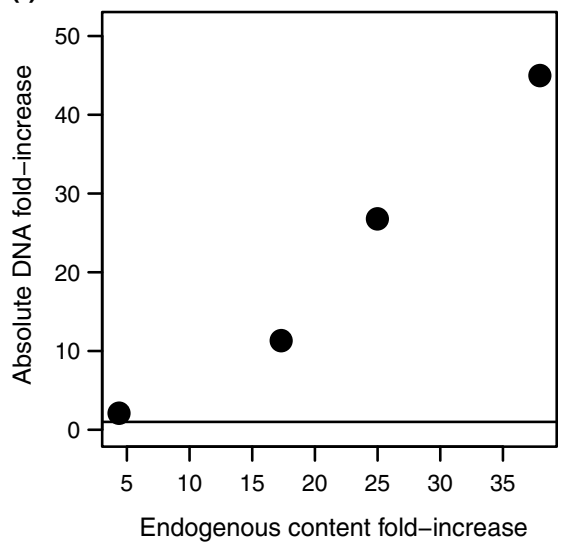

FIGURE 2 Comparisons of DNA obtained from the high-density otic capsule versus trabecular regions of petrous bones. (a) shows the fractions of endogenous DNA molecules recovered for each sample from the otic capsule (red) and the trabecular bone (green). The percentage of endogenous DNA content was determined as the number of mapping reads to the reference divided by the total number of reads after trimming. Graphs b-f compare, for each sample, the fold-increase proportion of endogenous molecules provided by the otic capsule, relative to trabecular bone ( $x$-axes), with another variable of interest ( $y$-axes): (b) Change in the proportion of endogenous molecules ( $x$-axis) versus change in the proportion of endogenous nucleotides ( $y$-axis). Diagonal line indicates a directly proportional relationship ( $x=y)$, as expected if fragment sizes obtained from each region are equal. (c) Change in the proportion of endogenous molecules ( $x$-axis) versus the change in mean fragment length of all recovered fragments (endogenous and contaminant, $y$-axis). (d) Change in the proportion of endogenous molecules ( $x$-axis) versus change in the proportion of deaminated cytosines at the terminal $5^{\prime}$ nucleotide. (e) Change in the proportion of endogenous molecules ( $x$-axis) versus change in the proportion of human contamination. (f) Change in the proportion of endogenous molecules $(x$-axis) versus change in the absolute mass of endogenous DNA obtained (calculated as the estimated endogenous fraction of total DNA obtained). In $c-f$, Horizontal lines $(y=1)$ indicate no change in that variable between otic capsule and trabecular treatments. Points positioned above and below this line represent an increase or decrease, respectively, in otic capsule versus trabecular sampling

lengths or single-stranded nick frequency associated with the outermost layer. This contrasts with patterns observed from petrous bones, where endogenous fragments obtained from the otic capsule were noticeably longer than those obtained from trabecular bone (Figure 2c). Comparisons of median fragment lengths of all molecules (endogenous and contaminant) obtained from the long bones did suggest an apparent species-specific effect: In cave bears, the median fragment length obtained from the outermost layer was slightly larger than that obtained from less dense bone regions, but for leopards this pattern is reversed (Figure 4d).

We investigated whether levels of cytosine deamination at the terminal $5^{\prime}$ end of DNA fragments sampled from the outermost layer showed any obvious difference from fragments obtained from trabecular bone or conventional sampling. The absolute number of mapped reads for some samples was too low to accurately measure the deamination pattern, so they were excluded from this comparison (Table 1). For the remaining samples, we found that, in all cases, DNA sampled from the outermost layer had lower levels of deamination than that sampled from trabecular bone (Figure 4e). In contrast, DNA from the outermost layer showed higher levels of deamination than that obtained using the conventional sampling method (Figure 4e). These patterns are also recovered when the alternative data processing pipeline is employed (Supporting Information Table S2). 


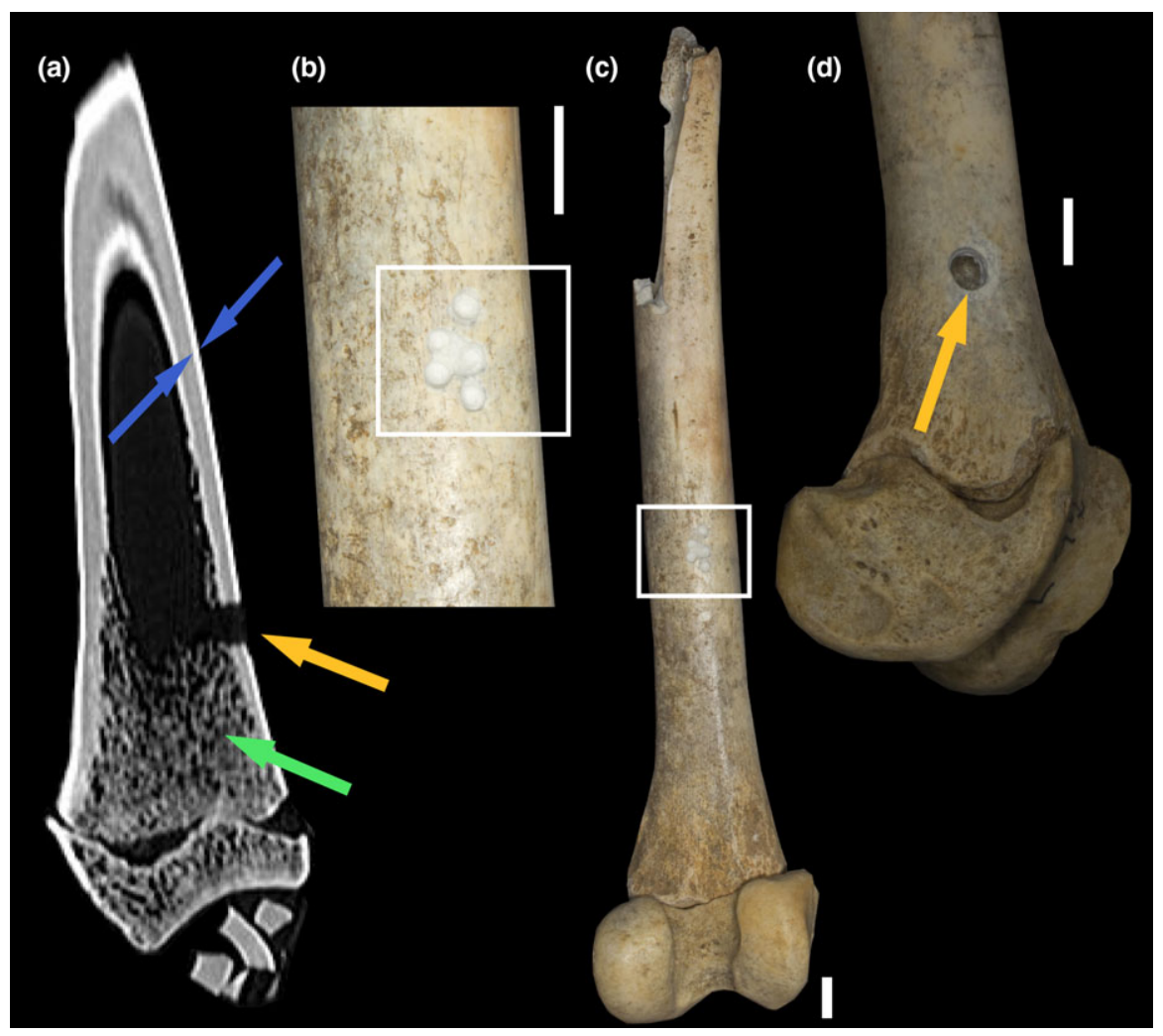

FIGURE 3 Long bone sampling. (a) CT scan image showing variation in bone density. The high-density outermost bone layer is clearly visible (blue arrows). The trabecular region is indicated by the green arrow. The location of conventional sampling is visible in the lower-right section of the CT scan (orange arrow). (b) Photograph showing the surface of the midshaft of the same bone, and the sampling location of the outermost layer, which was performed by drilling shallow holes into the surface of the bone (white square) according to the thickness of the outermost layer identified by the CT scan. (c) Photograph showing the complete bone. The white box indicates the sampling location of the outermost layer, which is barely visible at this magnification. (d) Conventional sampling of the same bone (orange arrow). After removal of the outermost bone surface, a small piece of the bone was removed using a core drill (orange arrow). All scale bars represent $1 \mathrm{~cm}$. A movie animation of the CT slices along the coronal section of this bone is shown in Supplementary Video S1

\section{DISCUSSION}

Although multiple methods exist for reducing the contaminant fraction of DNA obtained from ancient bones, optimization of the precise sampling location represents the simplest and potentially most effective approach. Our results show that, for both petrous and long bones, CT scanning and selection of the densest region of a bone results in on average much higher (factor of 21 for petrous and factor of eight for long bones) endogenous content compared to sampling trabecular bone or internal regions of compact bone using conventional methods. In some cases, CT-guided sampling also results in longer mean endogenous fragment lengths, reduced levels of cytosine deamination and an increase in absolute numbers of endogenous molecules. All these effects positively impact palaeogenomic studies using shotgun sequencing by decreasing the pernucleotide cost of endogenous data production. As hybridization capture success critically relies on the total amounts of endogenous DNA, which is often higher in the CT-selected regions, it is also likely that our screening approach will improve results obtained from hybridization capture when targeting ancient DNA. Additionally, minimally invasive approaches for sampling from largely complete skeletal elements, such as recently developed for sampling the petrous region from human skulls (Sirak et al., 2017), should also benefit from CT-guided sampling, which can inform the sampling strategy in a very precise way. For long bones, the result of sampling the outermost layer using the approach described here may also be considered visually preferable to conventional sampling methods (Figure 3b).

Our study also provides insights into the mechanisms of DNA survival in the bone matrix, which are not well understood (Campos et al., 2012; Lindahl, 1993; Schwarz et al., 2009). Screening of long bones revealed that the highest endogenous DNA concentrations are found in the densest outermost layer, which is comprised of cortical bone. Cortical bone has a primarily solid bone matrix containing osteons or Haversian systems (Currey, 2002). This highly dense structure has few internal open spaces, with the rods that form the Haversian system in particular made of highly compact bone, which our results suggest may lead to enhanced DNA preservation. This contrasts with trabecular bone, which lacks osteons and has a highly porous structure (Currey, 2002), potentially leading to greater exposure to external processes and contamination. A recent study of DNA damage and DNA loss in relation to time and other 
(a)

$\%$ endogenous DNA content

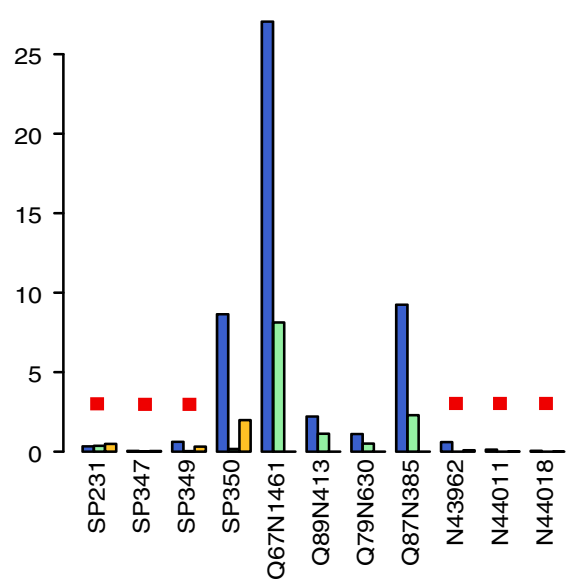

(d)

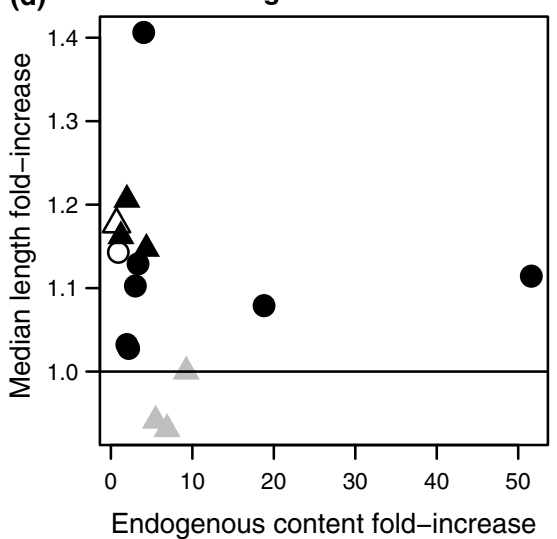

(b) \% endogenous DNA content

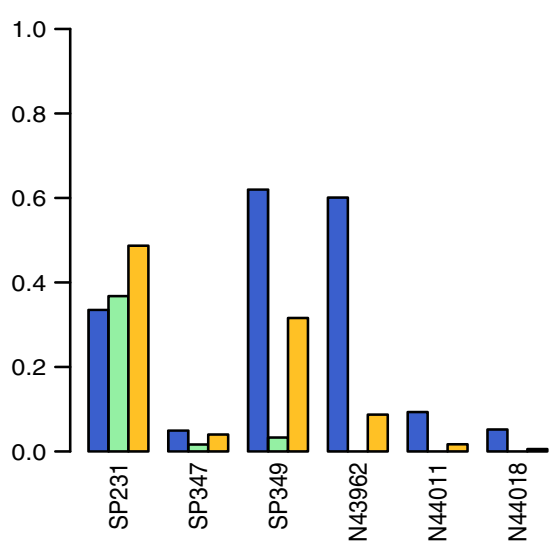

(e)

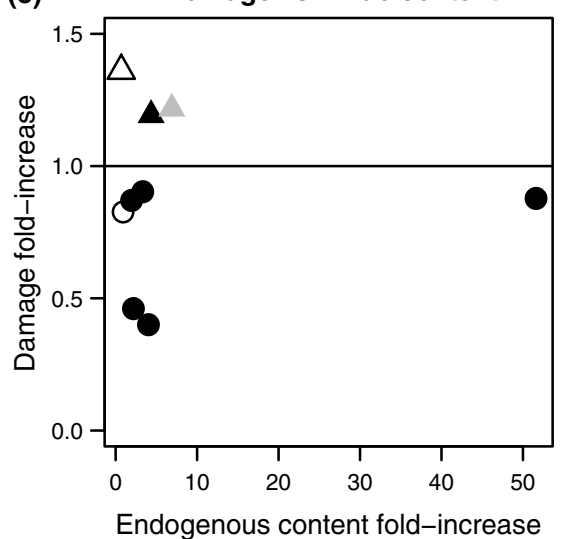

(c)

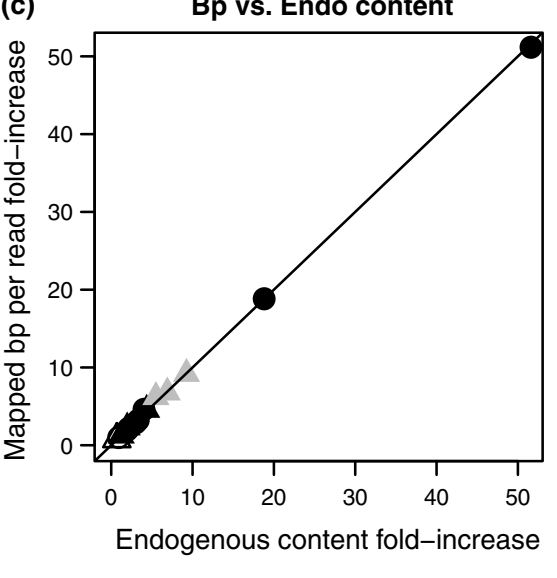

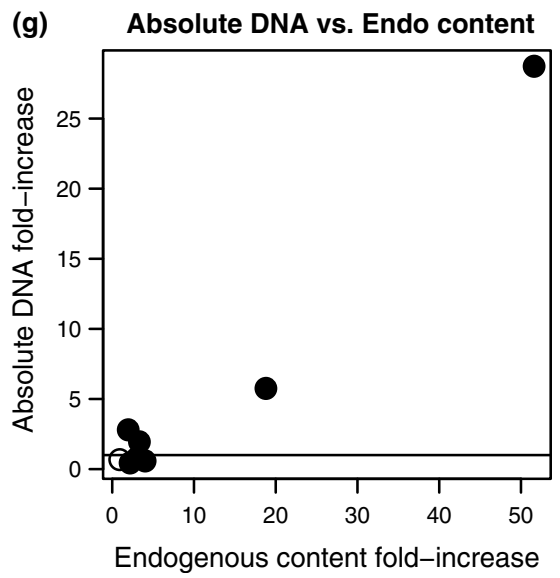

FIGURE 4 (a) Fractions of endogenous DNA molecules recovered for each sample from the outermost layer (blue), trabecular region (green) and conventional sampling (orange). (b) Rescaled graph for the samples with low endogenous content, indicated with a red square in panel a. (a-g) show comparisons of DNA obtained from the high-density outermost layer versus lower density region (trabecular and conventional treatments) of long bones. Details of plots are as described for Figure 2. Data points are categorized into cave bear (black) and leopard (grey), outermost layer vs. trabecular region (circles), outermost layer vs. conventional sampling method (triangles), and comparisons where the outermost layer provided an increase (filled shapes) or decrease (open shapes) in the proportion of endogenous molecules recovered in comparison with lower density internal bone regions. (f) For two samples, estimating the fold-increase ratio was not possible as no reads could be mapped to the human reference and are thus not included in this plot. (g) Total DNA yield was not measured for DNA obtained using the conventional sampling method. Note that, for comparisons of conventional sampling, data were also collected from the trabecular region of the same bone, resulting in two comparisons, respectively, with the outermost layer, which are not truly independent (see Table 1) 
environmental variables (Kistler, Ware, Smith, Collins, \& Allaby, 2017) proposed a similar hypothesis. The authors argued that DNA degradation proceeds more by leaching of DNA from bones rather than by fragmentation, but that certain bone structures and environments may be resistant to this bulk diffusion process "closed systems". Based on our results, both the otic capsule of petrous bones and the dense cortical bone of the outermost layer of long bones appear to fit this model.

The outermost layer of long bones is usually removed prior to sampling for ancient DNA in an effort to remove modern DNA contamination (Damgaard et al., 2015; Fortes et al., 2016; Hansen et al., 2017; Rohland \& Hofreiter, 2007). While this idea is intuitively plausible and observed in the majority of samples analysed here, we found that for some bones, this is not the case. This variability may be explained by the fact that contamination of ancient samples with modern DNA has been shown to depend heavily on the stage of a sample in the excavation and curation process (Gilbert, Hansen, Willerslev, Turner-Walker, \& Collins, 2006), as well as on variation in the permeability of different regions of the same bone to contaminant DNA molecules (Campos et al., 2012; Salamon, Tuross, Arensburg, \& Weiner, 2005). Moreover, the increased abundance of endogenous molecules may lead a reduced ratio of contaminant to endogenous DNA, even if absolute levels of contamination are increased. Overall, our results show that the assumption of excessive surface contamination may not apply for all samples. Given the potential increases in endogenous DNA recovery, sampling of the outermost layer using the method described here may thus represent an optimal approach for many studies on ancient long bones, particular when the modern contaminant sequences are sufficiently divergent to be excluded analytically.

Further interesting results are provided by the average fragment length and deamination patterns. The dense otic capsule of petrous bones consistently yielded longer total DNA fragments and lower deamination levels than trabecular bone. For long bones, the patterns are more complex. The average length of the total DNA fragments obtained from the outermost layer versus internal bone regions yielded opposite results for the two investigated species. This pattern may reflect a number of factors including species (Martiniakova, Grosskopf, Omelka, Vondrakova, \& Bauerova, 2006), sampling locality and the postexcavation environment. Deamination rates of DNA obtained from the outermost layer are intermediate between trabecular bone and internal cortical bone (obtained using conventional sampling). This suggests, in line with previous studies (Wanek \& Jakobus Rühli, 2016), that the CT scanning procedure has no substantial damaging effect on ancient DNA molecules, at least not above that normally observed for trabecular bone. Evidence of variable micropreservation of DNA between different regions of the same ancient bone has further implications for studies on the factors driving DNA degradation. For example, cytosine deamination has been frequently used for the validation of ancient DNA data authenticity (Gansauge \& Meyer, 2014; Ginolhac et al., 2011; Meyer et al., 2016; Sawyer, Krause, Guschanski, Savolainen, \& Pääbo, 2012), which our results show may vary by a factor of up to 1.36 for data sets obtained from different regions of the very same sample. Both cytosine deamination and DNA fragmentation have been used to study the decay kinetics of DNA over time (Allentoft et al., 2012; Kistler et al., 2017). Our results further suggest that sample micropreservation may represent an important factor to be taken into account for empirical investigations of these processes (Allentoft et al., 2012).

In conclusion, the positive correlation between bone density and both endogenous DNA content and DNA preservation has important implications for ancient DNA research. Using CT-guided sampling, we find further evidence supporting this relationship for the otic capsule of the petrous bones, as well as new evidence for the outermost layer, representing the densest region of long bones. Identifying new and improved methodologies for identifying and sampling high-density bone regions thus represents a future research direction with high potential in the study of palaeogenomes.

\section{ACKNOWLEDGEMENTS}

We thank Roberto Maria Micciche and Pietro Valenti for inspiring the use of CT scanning of ancient bones and Sina Baleka for bioinformatic support. This work was supported by ERC consolidator grant GeneFlow (310763 to $\mathrm{MH}$ ) and Klaus Tschira foundation project 00.298.2016.

\section{DATA ACCESSIBILITY}

Unprocessed Illumina sequencing data generated during this study have been uploaded to the European Nucleotide Archive (Accession no. PRJEB25493).

\section{AUTHOR CONTRIBUTIONS}

$\mathrm{MH}$ conceived the study; A.B. and J.L.A.P. designed experiments; G.F. and T.H. conducted CT scanning; F.A., J.G., M.P., K.H. and A.T. conducted laboratory work; J.G. invented the novel method for sampling the outermost layer; F.A. and N.B. analysed data; F.A., A.B., J.L.A.P. and M.H. interpreted results; A.B. and J.L.A.P. supervised laboratory work; A.B. supervised data analysis; G.R., N.J.C., S.C.M. and U.J. provided palaeontological samples and provided information of their context; A.B., F.A., J.L.A.P. and M.H. wrote the manuscript; F.A. prepared figures; M.H. obtained funding. All authors read and gave approval on the final version of the manuscript.

\section{ORCID}

Federica Alberti (iD http://orcid.org/0000-0002-1065-7490

\section{REFERENCES}

Allentoft, M. E., Collins, M., Harker, D., Haile, J., Oskam, C. L., Hale, M. L., ... Bunce, R. N. H. M. (2012). The half-life of DNA in bone: 
Measuring decay kinetics in 158 dated fossils. Proceedings Biological Sciences/The Royal Society, 279(1748), 4724-4733. https://doi.org/ 10.1098/rspb.2012.1745

Andrews, S. (2011). FastQ Screen [Online]. Retrieved from http://www. bioinformatics.babraham.ac.uk/projects/fastq_screen/.

Basler, N., Xenikoudakis, G., Westbury, M. V., Song, L., Sheng, G., \& Barlow, A. (2017). Reduction of the contaminant fraction of DNA obtained from an ancient giant panda bone. BMC Research Notes, 10 (1), 754. https://doi.org/10.1186/s13104-017-3061-3

Bennett, E. A., Massilani, D., Lizzo, G., Daligault, J., Geigl, E.-M., \& Grange, T. (2014). Library construction for ancient genomics: Single strand or double strand? BioTechniques, 56(6), 289-290, 292-96, 298, passim.

Briggs, A. W., Stenzel, U., Meyer, M., Krause, J., Kircher, M., \& Pääbo, S. (2010). Removal of deaminated cytosines and detection of in vivo methylation in ancient DNA. Nucleic Acids Research, 38(6), e87. https://doi.org/10.1093/nar/gkp1163

Campos, P. F., Craig, O. E., Turner-Walker, G., Peacock, E., Willerslev, E., Thomas, M., \& Gilbert, P. (2012). DNA in ancient bone - where is it located and how should we extract it? Annals of Anatomy - Anatomischer Anzeiger, 194(1), 7-16. https://doi.org/10.1016/j.aanat.2011. 07.003

Castellano, S., Parra, G., Sánchez-Quinto, F. A., Racimo, F., Kuhlwilm, M., Kircher, M., ... Paabo, S. (2014). Patterns of coding variation in the complete exomes of three neandertals. Proceedings of the National Academy of Sciences of the United States of America, 111(18), 66666671. https://doi.org/10.1073/pnas.1405138111

Cramer, L., Brix, A., Matin, E., Rühli, F., \& Hussein, K. (2017). Computed tomography-detected paleopathologies in ancient egyptian mummies. Current Problems in Diagnostic Radiology, 47, 225-232. https://doi. org/10.1067/j.cpradiol.2017.06.012

Currey, J. D. (2002). Structure and mechanics. Princeton, NJ: Princeton University Press.

Clio Der, S., Brotherton, P., Balanovsky, O., Templeton, J. E. L., Llamas, B., ... The Genographic Consortium (2014). Mitochondrial genome sequencing in mesolithic north east europe unearths a new sub-clade within the broadly distributed human haplogroup C1. PLoS ONE, 9(2), e87612. https://doi.org/10.1371/journal.pone.0087612

Dabney, J., Knapp, M., Glocke, I., Gansauge, M.-T., Weihmann, A., Nickel, B., ... Meyer, M. (2013). Complete mitochondrial genome sequence of a middle pleistocene cave bear reconstructed from ultrashort DNA fragments. Proceedings of the National Academy of Sciences of the United States of America, 110(39), 15758-15763. https://doi.org/10. 1073/pnas.1314445110

Damgaard, P. B., Margaryan, A., Schroeder, H., Orlando, L., Willerslev, E., \& Allentoft, M. E. (2015). Improving access to endogenous DNA in ancient bones and teeth. Scientific Reports, 5(June), 11184. https://d oi.org/10.1038/srep11184

Davis, M. P. A., van Dongen, S., Abreu-Goodger, C., Bartonicek, N., \& Enright, A. J. (2013). Kraken: A set of tools for quality control and analysis of high-throughput sequence data. Methods, 63(1), 41-49. https://doi.org/10.1016/j.ymeth.2013.06.027

Enk, J. M., Devault, A. M., Kuch, M., Murgha, Y. E., Rouillard, J.-M., \& Poinar, H. N. (2014). Ancient whole genome enrichment using baits built from modern DNA. Molecular Biology and Evolution, 31(5), 1292 1294. https://doi.org/10.1093/molbev/msu074

Fortes, G. G., Grandal-d'Anglade, A., Kolbe, B., Fernandes, D., Meleg, I. N., García-Vázquez, A., \& Barlow, A. (2016). Ancient DNA reveals differences in behaviour and sociality between brown bears and extinct cave bears. Molecular Ecology, 25(19), 4907-4918. https://doi.org/10. $1111 /$ mec. 13800

Gamba, C., Hanghøj, K., Gaunitz, C., Alfarhan, A. H., Alquraishi, S. A., AlRasheid, K. A. S., ... Orlando, L. (2016). Comparing the performance of three ancient DNA extraction methods for high-throughput sequencing. Molecular Ecology Resources, 16(2), 459-469. https://doi. org/10.1111/1755-0998.12470

Gamba, C., Jones, E. R., Teasdale, M. D., McLaughlin, R. L., Gonzalez-Fortes, G., Mattiangeli, V., ... Pinhasi, R. (2014). Genome flux and stasis in a five millennium transect of European prehistory. Nature Communications, 5(October), 5257. https://doi.org/10.1038/ncomms6257

Gansauge, M.-T., \& Meyer, M. (2013). Single-stranded DNA library preparation for the sequencing of ancient or damaged DNA. Nature Protocols, 8(4), 737-748. https://doi.org/10.1038/nprot.2013.038

Gansauge, M.-T., \& Meyer, M. (2014). Selective enrichment of damaged DNA molecules for ancient genome sequencing. Genome Research, 24 (9), 1543-1549. https://doi.org/10.1101/gr.174201.114

Gilbert, M. T. P., Hansen, A. J., Willerslev, E., Turner-Walker, G., \& Collins, M. (2006). Insights into the processes behind the contamination of degraded human teeth and bone Samples with exogenous sources of DNA. International Journal of Osteoarchaeology, 16(2), 156-164. https://doi.org/10.1002/oa.832

Ginolhac, A., Rasmussen, M., Thomas, M., Gilbert, P., Willerslev, E., \& Orlando, L. (2011). mapDamage: Testing for damage patterns in ancient DNA Sequences. Bioinformatics, 27(15), 2153-2155. https://d oi.org/10.1093/bioinformatics/btr347

Glocke, I., \& Meyer, M. (2017). Extending the spectrum of DNA sequences retrieved from ancient bones and teeth. Genome Research, 27(7), 1230-1237. https://doi.org/10.1101/gr.219675.116

Gnirke, A., Melnikov, A., Maguire, J., Rogov, P., LeProust, E. M., Brockman, W., ... Nusbaum, C. (2009). Solution hybrid selection with ultra-long oligonucleotides for massively parallel targeted sequencing. Nature Biotechnology, 27(2), 182-189. https://doi.org/10.1038/ nbt.1523

Griffin, L. R., Rawlinson, J. E., McDonald, H. G., \& Duncan, C. (2016). Mandibular osteopathy in a hagerman Horse, Equus Simplicidens (Equidae, Mammalia), from Hagerman Fossil Beds National Monument (Idaho, USA). International Journal of Paleopathology, 12(Supplement C), 41-45. https://doi.org/10.1016/j.ijpp.2015.11.002

Hansen, H. B., Damgaard, P. B., Margaryan, A., Stenderup, J., Lynnerup, N., Willerslev, E., \& Allentoft, M. E. (2017). Comparing ancient DNA preservation in petrous bone and tooth cementum. PLoS ONE, 12(1), e0170940. https://doi.org/10.1371/journal.pone.0170940

Hodges, E., Xuan, Z., Balija, V., Kramer, M., Molla, M. N., Smith, S. W., ... McCombie, W. R. (2007). Genome-wide in situ exon capture for selective resequencing. Nature Genetics, 39(12), 1522-1527. https://d oi.org/10.1038/ng.2007.42

lurino, D. A., Danti, M., Sala, S. W. D., \& Sardella, R. (2013). Modern techniques for ancient bones: Vertebrate palaeontology and medical CT analysis. Boll. Soc. Paleontol. ItalY, 52(3), 14.

Jónsson, H., Ginolhac, A., Schubert, M., Johnson, P. L. F., \& Orlando, L. (2013). mapDamage2.0: Fast approximate bayesian estimates of ancient DNA damage parameters. Bioinformatics, 29(13), 1682-1684. https://doi.org/10.1093/bioinformatics/btt193

Kappelman, J., Ketcham, R. A., Pearce, S., Todd, L., Akins, W., Colbert, M. W., ... Witzel, A. (2016). Perimortem fractures in lucy suggest mortality from fall out of tall tree. Nature, 537(7621), 503-507. https://d oi.org/10.1038/nature19332

Kistler, L., Ware, R., Smith, O., Collins, M., \& Allaby, R. G. (2017). A new model for ancient DNA decay based on paleogenomic meta-analysis. Nucleic Acids Research, 45, 6310-6320. https://doi.org/10.1093/nar/ gkx361 https://doi.org/10.1093/nar/gkx361

Korlević, P., Gerber, T., Gansauge, M.-T., Hajdinjak, M., Nagel, S., AximuPetri, A., \& Meyer, M. (2015). Reducing microbial and human contamination in DNA extractions from ancient bones and teeth. BioTechniques, 59(2), 87-93.

Lautenschlager, S. (2016). Reconstructing the past: Methods and techniques for the digital restoration of fossils. Royal Society Open Science, 3(10), 160342. https://doi.org/10.1098/rsos.160342 
Li, H., \& Durbin, R. (2009). Fast and accurate short read alignment with burrows-wheeler transform. Bioinformatics, 25(14), 1754-1760. https://doi.org/10.1093/bioinformatics/btp324

Li, H., Handsaker, B., Wysoker, A., Fennell, T., Ruan, J., Homer, N., ... 1000 Genome Project Data Processing Subgroup. (2009). The Sequence alignment/map format and SAMtools. Bioinformatics, 25 (16), 2078-2079. https://doi.org/10.1093/bioinformatics/btp352

Lindahl, T. (1993). Instability and decay of the primary structure of DNA. Nature, 362(6422), 709-715. https://doi.org/10.1038/362709a0

Martin, M. (2011). Cutadapt removes adapter sequences from highthroughput sequencing reads. EMBnet.journal, 17(1), 10-12. https://d oi.org/10.14806/ej.17.1.200

Martiniakova, M., Grosskopf, B., Omelka, R., Vondrakova, M., \& Bauerova, M. (2006). Differences among species in compact bone tissue microstructure of mammalian skeleton: Use of a discriminant function analysis for species identification. Journal of forensic sciences, 51(6), 1235-1239. https://doi.org/10.1111/j.1556-4029.2006.00260.x

Meyer, M., Arsuaga, J.-L., de Filippo, C., Nagel, S., Aximu-Petri, A., Nickel, B., ... Pääbo, S. (2016). Nuclear DNA sequences from the middle pleistocene sima de los huesos hominins. Nature, 531(7595), 504 507. https://doi.org/10.1038/nature17405

Noonan, J. P., Hofreiter, M., Smith, D., Priest, J. R., Rohland, N., Rabeder, G., ... Rubin, E. M. (2005). Genomic sequencing of pleistocene cave bears. Science, 309(5734), 597-599. https://doi.org/10.1126/science.1113485

Orlando, L., Ginolhac, A., Zhang, G., Froese, D., Albrechtsen, A., Stiller, M., ... Willerslev, W. (2013). Recalibrating equus evolution using the genome sequence of an early middle Pleistocene horse. Nature, 499 (7456), 74-78. https://doi.org/10.1038/nature12323

Paijmans, J. L. A., Baleka, S., Henneberger, K., Taron, U. H., Trinks, A., Westbury, M. V., \& Barlow, A. (2017). Sequencing single-stranded libraries on the illumina NEXTSEQ 500 Platform. arXiv [q-bio.OT]. arXiv. http://arxiv.org/abs/1711.11004.

Paijmans, J. L. A., Fickel, J., Courtiol, A., Hofreiter, M., \& Förster, D. W. (2016). Impact of enrichment conditions on cross-species capture of fresh and degraded DNA. Molecular Ecology Resources, 16(1), 42-55. https://doi.org/10.1111/1755-0998.12420

Pinhasi, R., Fernandes, D., Sirak, K., Novak, M., Connell, S., Alpaslan-Roodenberg, S., ... Hofreiter, M. (2015). Optimal ancient DNA yields from the inner ear part of the human petrous bone. PLoS ONE, 10(6), e0129102. https://doi.org/10.1371/journal.pone.0129102

Rohland, N., \& Hofreiter, M. (2007). Ancient DNA extraction from bones and teeth. Nature Protocols, 2(7), 1756-1762. https://doi.org/10. 1038/nprot.2007.247
Salamon, M., Tuross, N., Arensburg, B., \& Weiner, S. (2005). Relatively well preserved DNA Is present in the crystal aggregates of fossil bones. Proceedings of the National Academy of Sciences of the United States of America, 102(39), 13783-13788. https://doi.org/10.1073/ pnas.0503718102

Sawyer, S., Krause, J., Guschanski, K., Savolainen, V., \& Pääbo, S. (2012). emporal patterns of nucleotide misincorporations and DNA fragmentation in ancient DNA. PLoS ONE, 7(3), e34131. https://doi.org/10. 1371/journal.pone.0034131

Schwarz, C., Debruyne, R., Kuch, M., McNally, E., Schwarcz, H., Aubrey, A. D., ... Poinar, H. (2009). New insights from old bones: DNA preservation and degradation in permafrost preserved mammoth remains. Nucleic Acids Research, 37(10), 3215-3229. https://doi.org/ 10.1093/nar/gkp159

Sirak, K. A., Fernandes, D. M., Cheronet, O., Novak, M., Gamarra, B., Balassa, T., ... Pinhasi, R. (2017). A minimally-invasive method for sampling human petrous bones from the cranial base for ancient DNA analysis. BioTechniques, 62(6), 283-289.

Väre, T., Niinimäki, J., Junno, J.-A., Núñez, M., Niinimäki, S., \& Niskanen, M. (2016). Suspected tuberculosis in an early 17th-century northern Finnish mummy - A computed tomography case study. International Journal of Paleopathology, 14(Supplement C), 69-73. https://doi.org/ 10.1016/j.ijpp.2016.05.009

Wanek, J., \& Jakobus Rühli, F. (2016). Risk to fragmented DNA in dry, wet, and frozen states from computed tomography: A comparative theoretical study. Radiation and Environmental Biophysics, 55(2), 229241. https://doi.org/10.1007/s00411-016-0637-6

\section{SUPPORTING INFORMATION}

Additional supporting information may be found online in the Supporting Information section at the end of the article.

How to cite this article: Alberti F, Gonzalez J, Paijmans JLA, et al. Optimized DNA sampling of ancient bones using Computed Tomography scans. Mol Ecol Resour. 2018;00:1-13. https://doi.org/10.1111/1755-0998.12911 